УДК 624.121:550.47:556.54

МОДЕЛЬНОЕ ИССЛЕДОВАНИЕ ПРОЦЕССОВ ДЕГРАДАЦИИ

ВЫЛИТОЙ НА ПОВЕРХНОСТЬ МОРЯ СЫРОЙ НЕФТИ

\title{
MODEL STUDIES OF THE DEGRADATION OF CRUDE OIL SPILLED \\ ON THE SEA SURFACE
}

Х.Г. Асадов, Р.Ш. Маммадли

\section{Национальное аэрокосмическое агентство, г. Баку, Азербайджанская Республика \\ Hikmat H. Asadov, Rashad Sh. Mammadli
Aerospace Agency, Baku, Republic of Azerbaijan
e-mail: asadzade@rambler.ru

Аннотация. Статья посвящена модельному исследованию процессов деградации вылитой на поверхность моря сырой нефти.

Возрастающая потребность в углеводородных источниках энергии в современном мире диктует необходимость развития и расширения методов и масштабов транспортировки нефти газа. Транспортировка сырой нефти по морю на танкерах или по трубопроводу всегда сопряжена с риском техногенных катастроф, результатом которых неизбежно является вылив нефти в моря или океаны, что наносит существенный вред морской флоре и фауне и является крайне нежелательным для морской экологии. Вышеуказанное доказывает важность и актуальность модельных исследований процессов трансформации вылившейся нефтяной массы в водную среду морей и океанов. Находящаяся на поверхности моря нефтяная масса подвергается деградации под воздействием различных физико-биологических факторов, приводящих к изменению плотности и вязкости сырой нефти. Очевидно, что исследование деградационных 
процессов нефти важно для планирования мер противодействия пагубным процессам в зонах техногенных катастроф.

Предложена новая схема упрощенной оптимизационной модели процесса деградации нефти на морской поверхности. На основе предложенной схемы разработана упрощенная оптимизационная модель деградации нефти на морской поверхности. Установлена оптимальная взаимосвязь временной зависимости скорости ветра и фракционной величины улетучивания нефти, при которой достигается минимальная фракционная величина растворенной в воде нефти.

Abstract. The article is devoted to a model study of the degradation of crude oil poured onto the surface of the sea.

The increasing demand for hydrocarbon energy sources in the modern world dictates the need to develop and expand the methods and scale of oil gas transportation. Crude oil transportation by sea in tankers or by pipeline is always associated with the risk of technological disasters, the result of which is inevitably the spill of oil into the seas or oceans, which causes significant harm to the marine flora and fauna and is extremely undesirable for marine ecology. The above proves the importance and relevance of model studies of the processes of spilled oil mass transformation into the seas and oceans aquatic environment. The oil mass located on the sea surface undergoes degradation under the various physical and biological factors influence leading to a change in crude oil density and viscosity. Obviously, the study of oil degradation processes is important for planning measures to counter harmful processes in areas of technological disasters.

A new scheme of a simplified optimization model of the oil degradation process on the sea surface is proposed. Based on the proposed scheme, a simplified optimization model for oil degradation on the sea surface has been developed. The optimal relationship between the time dependence of the wind speed and the fractional value of the volatilization of oil is established, at which the minimum fractional value of oil dissolved in water is achieved. 
Ключевые слова: деградация; углеводороды; моделирование; оптимизация; фракционный объем

Key words: degradation; hydrocarbons; modeling; optimization; fraction volume

\section{Введение}

Возрастающая потребность в углеводородных источниках энергии в современном мире диктует необходимость развития и расширения методов и масштабов транспортировки нефти газа. Транспортировка сырой нефти по морю на танкерах или по трубопроводу всегда сопряжена с риском техногенных катастроф, результатом которых неизбежно является вылив нефти в моря или океаны, что наносит существенный вред морской флоре и фауне и является крайне нежелательным для морской экологии. Вышеуказанное доказывает важность и актуальность модельных исследований процессов трансформации вылившейся нефтяной массы в водную среду морей и океанов. Находящаяся на поверхности моря нефтяная масса подвергается деградации под воздействием различных физико-биологических факторов, приводящих к изменению плотности и вязкости сырой нефти [1-3]. Очевидно, что исследование деградационных процессов нефти важно для планирования мер противодействия пагубным процессам в зонах техногенных катастроф.

\section{Цель и задачи исследований}

К деградационным процессам, происходящим с свежевылитой нефтяной пленкой на морской поверхности, относятся следующие:

- распространение на поверхности;

- испарение;

- растворение;

- дисперсия; 
- эмульсификация и др.

Указанные процессы являются комплексными и происходят во времени параллельно. Вместе с тем создание единой математической модели, учитывающей все вышеуказанные процессы, является сложнейшей задачей экологического моделирования, и внастоящей статье мы преследуем цель разработки упрощенной модели деградащии нефти, в которой учитываются только первые три процесса из числа вышеуказанных, т.е. распространение на поверхности, испарение и растворение.

\section{Существующие модели рассматриваемых деградационных процессов нефти}

Согласно [4] распространение нефтяного пятна на поверхности моря происходит в три этапа:

- инерциально-гравитационный этап;

- гравитационно-вязкостный этап;

- вязкостно-поверхностное напряжение.

Наиболее длительным является гравитационно-вязкостный этап, в результате которого площадь нефтяного пятна $A$ определяется по следующему дифференциальному уравнению [4]:

$$
\frac{d A}{d t}=\frac{k_{s p} \cdot V^{\frac{4}{3}}}{A},
$$

где $V$ - обьем нефти в момент времени $t\left(\mathrm{~m}^{3}\right)$;

$k_{s p}$ - постоянная испарения $\left(150 \mathrm{c}^{-1}\right)$.

Процесс испарения является важнейшим механизмом удаления нефти с поверхности моря. Скорость испарения определяется по уравнению [5]:

$$
\frac{d F_{e}}{d t}=\frac{\kappa_{e v p} \cdot A}{V_{0}} \cdot\left[a-\frac{\left(T_{0}+T_{g}+F_{e}\right)}{T_{o i l}}\right],
$$


где $F_{e}$ - объем испарившейся фракции нефти (\%);

$\kappa_{\text {evp }}$ - коэффициент массо-переноса процесса испарения (м/с);

$a, b-$ постоянные процесса испарения;

$T_{0}$ - исходная температура кипения нефти;

$T_{g}$ - градиент кривой дистилляции нефти (К);

$T_{o i l}$ - температура вылитой нефти, принимаемая равной температуре поверхностных вод.

При этом $\kappa_{\text {evp }}$ определяется по следующей формуле:

$$
\kappa_{\text {evp }}=2,5 \cdot 10^{-3} W^{0,78} \text {, }
$$

где $W$ - скорость ветра на высоте 10 м (м/с).

Показатели $T_{0}$ и $T_{g}$ определяются как

$$
\begin{aligned}
& T_{0}=532,98-3,125 A P I, \\
& T_{g}=985,62-13,597 A P I,
\end{aligned}
$$

где $A P I$ - показатель удельной плотности нефти по шкале Американского

Института Нефти API.

Очевидно, что расстворение нефти в морской воде приводит к увеличению ее токсичности. Количество растворенной в воде нефти определяется следующим уравнением [6]:

$$
\frac{d F_{d}}{d t}=K_{d i s s} A\left(\frac{S}{1000 \rho_{\text {oil }}}\right)
$$

где

$$
S=S_{0} \cdot \exp \left(-12,0 \cdot F_{e}\right),
$$

где $F_{d}$ - фракционный обьем расстворенной в морской воде нефти (\%);

$K_{\text {diss }}-$ коэффициент масса-переноса процесса расстворения (м/с);

$S$ - расстворимость нефти в момент $t\left(\Gamma / \mathrm{M}^{3}\right)$;

$S_{0}-$ исходная растворимость нефти в воде $\left(\Gamma / \mathrm{M}^{3}\right)$;

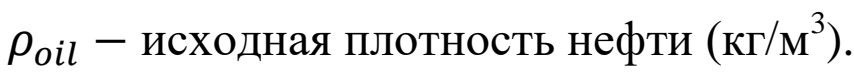




\section{Схема упрощенной оптимизационной модели процесса деградации} нефти на морской поверхности

В отличие от вышеприведенных модельных соотношений различных деградационных процессов в настоящей работе ставится цеель разработки единой оптимизационной модели процесса деградация нефти на морской поверхности.

Суть оптимизационной модели формально можно изложить следующим образом. Допустим, что имеется множество $\mathrm{X}$ взаимосвязанных частных процессов, характерных для деградации нефти, определяемое как

$$
\mathrm{X}=\left\{x_{1}(t) ; x_{2}(t) ; x_{3}(t) ;\right\}
$$

$x_{i}(t) ; i=\overline{1,3}$;- $\quad$ частные процессы, определяющие общий процесс деградации нефти.

Требуется определить оптимальное соотношение между процессами $x_{2}(t)$ и $x_{3}(t)$, при котором может быть достигнуто минимальное значение следующего интегрального показателя:

$$
\mathrm{X}_{1 \text { int }}=\int_{T_{0}}^{T_{f}} x_{1}(t) d t
$$

где $T_{0}$ - фиксированный момент вылива нефти;

$T_{f}$ - фиксированный момент оценки.

С учетом факта повышения токсичности морских вод из-за растворения нефти в воде вышеуказанные частные процессы определим как:

$$
\begin{aligned}
& x_{1}(t)=F_{d}(t) ; \\
& x_{2}(t)=F_{\mathrm{e}}(t) ; \\
& x_{3}(t)=W(t) .
\end{aligned}
$$

Рассмотрим вопросы оптимизации предлагаемой упрощенной модели. 
Разработка упрощуенной оптимизащионной модели деградащии нефти

С учетом выражений (6) и (7) напишем

$$
\frac{d F_{d}}{d t}=\frac{K_{\text {diss }} A \cdot S_{0} \exp \left(-12,0 \cdot F_{e}\right)}{1000 \rho_{\text {oil }}} .
$$

Интегрируя (13) с учетом $F_{e}=F_{e}(t), \mathrm{A}=\mathrm{A}(t)$, получим

$$
F_{d}=\int_{0}^{T_{f}} \frac{K_{d i s s} A \cdot S_{0} \exp \left(-12,0 \cdot F_{e}\right)}{1000 \rho_{\text {oil }}} d t
$$

Из выражения (2) с учетом $\mathrm{A}=\mathrm{A}(t)$ получим

$$
A(t)=\frac{V_{0}}{K_{e v p}} \cdot\left(\frac{d F_{e}}{d}\right) \cdot\left[\frac{1}{a-\frac{b\left(T_{0}+T_{g} \cdot F_{e}\right)}{T_{o i l}}}\right] .
$$

С учетом выражений (14) и (15) имеем

$$
F_{d}=\int_{0}^{T_{f}}\left\{\frac{K_{\text {diss }} \cdot S_{0} \cdot V_{0} \cdot\left(\frac{d F_{e}}{d}\right) \cdot \exp \left(-12,6 \cdot F_{e}(t)\right)}{1000 \rho_{\text {oil }} \cdot K_{\text {evp }}\left[a-\frac{b\left(T_{0}+T_{g} \cdot F_{e}\right)}{T_{\text {oil }}}\right]}\right\} d t .
$$

Примем следующее обозначение

$$
D\left(F_{e}(t), \frac{d F_{e}}{d}\right)=\left\{\frac{K_{d i s s} \cdot S_{0} \cdot V_{0} \cdot\left(\frac{d F_{e}}{d}\right) \cdot \exp \left(-12,6 \cdot F_{e}(t)\right)}{1000 \rho_{\text {oil }} \cdot K_{e v p}\left[a-\frac{b\left(T_{0}+T_{g} \cdot F_{e}\right)}{T_{\text {oil }}}\right]}\right\} .
$$

С учетом выражений (3) и (17) функционал (16) напишем следующим образом:

$$
F_{d}=\int_{0}^{T_{f}} \frac{D\left(F_{e}(t), \frac{d F_{e}}{d}\right)}{2,5 \cdot 10^{-3} W(t)^{0,78}} d t
$$


Учитывая случайный характер временного изменения скорости ветра, примем следующую модель $W(t)$ :

$$
\int_{0}^{T_{f}} W(t) d t=\mathrm{C} ; \quad \mathrm{C}=\text { const } .
$$

На основе выражений (18) и (19) составим полный функционал безусловной вариационной оптимизации:

$$
F_{d}=\int_{0}^{T_{f}} \frac{D\left(F_{e}(t), \frac{d F_{e}}{d}\right)}{2,5 \cdot 10^{-3} W(t)^{0,78}} d t+\lambda\left[\int_{0}^{T_{f}} W(t) d t-\mathrm{C}\right] .
$$

Вычислим оптимальную функцию $W(t)$, при которой $F_{d} \rightarrow$ min.

Согласно [7] решение данной задачи должно удовлетворить условию

$$
\frac{d\left\{\frac{D\left(F_{e}(t), \frac{d F_{e}}{d}\right)}{2,5 \cdot 10^{-3} W(t)^{0,78}} d t+\lambda\left[\int_{0}^{T_{f}} W(t)-C\right]\right\}}{d W(t)}=0 .
$$

Из выражения (21) получим

$$
-0,78 \cdot 400 \cdot D\left(F_{e}(t), \frac{d F_{e}}{d}\right) W(t)^{-1,78}+\lambda=0
$$

Из выражения (22) находим

$$
W(t)=\frac{0,78 \cdot 400 \cdot D\left(F_{e}(t), \frac{d F_{e}}{d}\right)}{\lambda} .
$$

С учетом выражений (19) и (23) можно вычислить значение $\lambda$, результат такого вычисления обозначим как $\lambda_{0}$ :

$$
\lambda_{0}=\int_{0}^{T_{f}} \frac{0,78 \cdot 400 \cdot D\left(F_{e}(t), \frac{d F_{e}}{d}\right)}{\mathrm{C}} d(t) .
$$

С учетом выражений (19) и (23) получим 


$$
W(t)=\frac{D\left(F_{e}(t), \frac{d F_{e}}{d}\right)}{\int_{0}^{T_{f}} D\left(F_{e}(t), \frac{d F_{e}}{d}\right) d(t)} .
$$

Используя выражение (22), можно показать, что при решении (25) целевой функционал (20) достигает минимума. Для этого достаточно вычислить вторую призводную интегранта в $(20)$ по $W(t)$ и убедиться, что она положительна.

\section{Заключение и выводы}

Предлагаемая упрощенная оптимизационная модель деградационного процесса свежевылитой нефти на поверхности моря учитывает влияние таких процессов, как растворение нефти в воде, испарение нефтяных фракций и скорость ветра.

Получена аналитическая зависимость $W(t)$ от таких показателей, как $F_{e}(t)$ и $F_{e}^{\prime}(t)$, при которых фракционная величина растворенной в воде нефти достигает минимальной величины, что равносильно достижению минимальной токсичности морских вод в рамках построенной модели деградации нефти на поверхности морских вод.

\section{Выводы}

1. Предложена новая схема упрощенной оптимизационной модели процесса деградации нефти на морской поверхности.

2. На основе предложенной схемы разработана упрощенная оптимизационная модель деградации нефти на морской поверхности.

3. Определена оптимальная взаимосвязь временной зависимости скорости ветра и фракционной величины испарения нефти, при которой достигается минимальная фракционная величина растворенной в воде нефти. 


\section{Список используемых источников}

1. Fingas V.F. Water-in-Oil Emulsions: Formation and Prediction // Journal of Petroleum Science Research. 2014. Vol. 3. P. 38-49. DOI: 10.14355/jpsr.2014.0301.04.

2. Azevedo A., Oliveira A., Fortunato A.B., Zhang J., Baptista A.M., A Cross-Scale Numerical Modeling System for Management Support of Oil Spill Accidents // Marine Pollution Bulletin. 2014. Vol. 80. No. 1-2. P. 132-147. DOI: 10.1016/j.marpolbul.2014.01.028.

3. Lehr W.J. Review of Modeling Procedures for Oil Spill Weathering Behavior // Advances in Ecological Sciences. 2001. No. 9. P. 51-90.

4. Sebastiao P., Soares C.G., Modeling the Fate Oil Spills at Sea // Spill Science \& Technology. 1995. Vol. 2. No. 2. P. 121-131.

5. Stiver W., Mackay D. Evaporation Rate of Spills of Hydrocarbons and Petroleum Mixtures // Environmental Science \& Technology. 1984. Vol. 18. No. 11. P. 834-840.

6. Shen H.T., Yapa P.D., Wang D.S., Yang X.Q. A Mathematical Model for Oil Slick Transport and Mixing in Rivers. Hanover: Cold Regions Research and Engineering, 1993. 78 p.

7. Эльсгольц Л.Э. Дифференциальные уравнения и вариационное исчисление. М.: Наука, 1974. 472 с.

\section{References}

1. Fingas V.F. Water-in-Oil Emulsions: Formation and Prediction. Journal of Petroleum Science Research, 2014, Vol. 3, pp. 38-49. DOI: 10.14355/jpsr.2014.0301.04.

2. Azevedo A., Oliveira A., Fortunato A.B., Zhang J., Baptista A.M. A Cross-Scale Numerical Modeling System for Management Support of Oil Spill Accidents. Marine Pollution Bulletin, 2014, Vol. 80, No. 1-2, pp. 132-147. DOI: 10.1016/j.marpolbul.2014.01.028. 
3. Lehr W.J. Review of Modeling Procedures for Oil Spill Weathering Behavior. Advances in Ecological Sciences, 2001, No. 9, pp. 51-90.

4. Sebastiao P., Soares C.G. Modeling the Fate Oil Spills at Sea. Spill Science \& Technology, 1995, Vol. 2, No. 2, pp. 121-131.

5. Stiver W., Mackay D. Evaporation Rate of Spills of Hydrocarbons and Petroleum Mixtures. Environmental Science \& Technology, 1984, Vol. 18, No. 11, pp. 834-840.

6. Shen H.T., Yapa P.D., Wang D.S., Yang X.Q., A Mathematical Model for Oil Slick Transport and Mixing in Rivers. Hanover, Cold Regions Research and Engineering, 1993. 78 p.

7. Elsgolts L.E. Differentsial'nye uravneniya $i$ variatsionnoe ischislenie [Differential Equations and Calculus of Variations]. Moscow, Nauka Publ., 1974. 472 p. [in Russian].

\section{Сведения об авторах}

\section{About the authors}

Асадов Хикмет Гамид оглы, д-р техн. наук, профессор, начальник отдела НИИ Аэрокосмической информатики Национального аэрокосмического агентства, г. Баку, Азербайджанская Республика

Hikmat H. Asadov, Doctor of Engineering Sciences, Professor, Head of Department, Research Institute of Aerospace Informatics, National Aerospace Agency, Baku, Azerbaijan Republic

e-mail: asadzade@rambler.ru

Маммадли Рашад Шохрат оглу, докторант Национального аэрокосмического агентства, г. Баку, Азербайджанская Республика

Rashad Sh. Mammadli, Doctorant of National Aerospace Agency, Baku, Azerbaijan Republic 